\title{
CATEGORICAL HOMOTOPY AND FIBRATIONS
}

\author{
BY \\ F.-W. BAUER AND J. DUGUNDJI( $\left.{ }^{1}\right)$
}

Let $\mathscr{K}$ be an arbitrary category, and let $\mathfrak{M}$ be any family of its morphisms. It is known [3] that there is a category $\mathscr{K} / \mathfrak{M}$ (the Gabriel-Zisman "category of fractions of $\mathscr{K}$ by $\mathfrak{M}$ ") having the same objects as $\mathscr{K}$, and a covariant functor $\eta: \mathscr{K} \rightarrow \mathscr{K} / \mathfrak{M}$ which is the identity on objects, such that $\eta(f)$ is invertible in $\mathscr{K} / \mathfrak{M}$ for each $f \in \mathfrak{M}$.

We will use each class $\mathfrak{M}$ to determine a notion of homotopy in $\mathscr{K}$, by defining two morphisms $f, g$ to be $\mathfrak{M}$-homotopic if $\eta(f)=\eta(g)$. This notion has the usual properties expected of a homotopy notion; moreover, in the category Top of topological spaces and continuous maps, suitable choices of $\mathfrak{M}$ (for example, $\mathfrak{M}=$ all homotopy equivalences) reveal $\mathfrak{M}$-homotopy equivalent to the usual notion of homotopy.

Each class $\mathfrak{M}$ determines a notion of fibration in $\mathscr{K}$. In the category Top, a suitable choice of $\mathfrak{M}$ determines both the usual homotopy and the Hurewicz fibrations; however, different classes $\mathfrak{M}$ may yield the usual notion of homotopy and distinct notions of fibration. More generally, in an arbitrary category $\mathscr{K}$, it is the given class $\mathfrak{M}$ itself, rather than the homotopy notion induced by $\mathfrak{M}$, that determines the concept of fibration; from this viewpoint, it turns out, surprisingly, that the notion of a Hurewicz fibration is not a homotopy notion. By "reversing arrows," $\mathfrak{M}$ determines also a concept of cofibration; and again there is a splitting: in fact, two classes may determine the same notion of homotopy but distinct notions of cofibration.

In the last section, we introduce the concept of a weak $\mathfrak{M}$-fibration. This notion does not, in general, possess all the advantages of the previous one; however, it reduces to the previous notion under suitable restrictions on the class $\mathfrak{M}$. Moreover, there are classes, $\mathfrak{M}, \mathfrak{N}$ in the category Top such that $\{$ weak $\mathfrak{M}$-fibrations $=$ $\{$ Hurewicz fibrations $\}$ and $\{$ weak $\mathfrak{R}$-fibrations $\}=\{$ Dold fibrations $\}$.

Each covariant functor $\Phi: \mathscr{K} \rightarrow \mathscr{L}$ determines a $\Phi$-homotopy in $\mathscr{K}$, by choosing $\mathfrak{M}=\{f \mid \Phi(f)$ is invertible $\}$. Most of the homotopy notions encountered in various categories turn out to stem from this general construction. For example: if $\mathscr{G}^{z}$ is the category of graded groups and degree zero homomorphisms, and if $\pi(X)$ is the total homotopy of a space $X$, then in the category $\mathscr{K}$ of spaces dominated by $\mathrm{CW}$-complexes the functor $\pi: \mathscr{K} \rightarrow \mathscr{G}^{Z}$ determines the usual notion of homotopy;

Received by the editors May 9, 1968.

( $\left.{ }^{1}\right)$ This research was partially supported by an NSF grant. 
if $\mathscr{K}$ is the category of Kan-complexes, then $\pi$-homotopy is the same as Kanhomotopy; and if $H: \mathscr{K} \rightarrow \mathscr{G}^{z}$ is the total homology functor on the category of chain-complexes, then $H$-homotopy is the usual chain-homotopy.

In the Appendix, we give a construction of $\mathscr{K} / \mathfrak{M}$ that differs slightly from that of Gabriel-Zisman, and contains slightly more detail; one advantage of this procedure is that some results in [3] are seen to hold without the additional requirement that “ $\mathfrak{M}$ admits a calculus of left fractions".

1. The quotient category $\mathscr{K} / \mathfrak{M}$. Let $\mathscr{K}$ be any category, and let $\mathfrak{M}$ be any family of its morphisms. By a quotient category we shall mean a pair $(\mathscr{K} / \mathfrak{M}$, $\eta)$, where $\mathscr{K} / \mathfrak{M}$ is a category with the same objects as $\mathscr{K}$ and $\eta: \mathscr{K} \rightarrow \mathscr{K} / \mathfrak{M}$ is a covariant functor that preserves objects, having the following two properties:

C1. If $\alpha \in \mathfrak{M}$, then $\eta(\alpha)$ is invertible in $\mathscr{K} / \mathfrak{M}$.

C2. [Universality]: If $T: \mathscr{K} \rightarrow \mathscr{L}$ is any covariant functor to any category $\mathscr{L}$ such that $T(\alpha)$ is invertible for each $\alpha \in \mathfrak{M}$, then there exists a unique covariant functor $\Delta: \mathscr{K} / \mathfrak{M} \rightarrow \mathscr{L}$ such that $T=\Delta \circ \eta$.

1.1. THEOREM. Let $\mathscr{K}$ be any category, and let $\mathfrak{M}$ be any family of its morphisms. Then a quotient category $(\mathscr{K} / \mathfrak{M}, \eta)$ exists.

This theorem is mentioned in [3]. However, no detailed construction of $(\mathscr{K} / \mathfrak{M}, \eta)$ having the generality we require appears in the literature, so we will give a proof of 1.1 in the Appendix. It follows directly from our construction, and we will need this in the sequel, that

1.2. Proposition. Each morphism $G$ in $\mathscr{K} / \mathfrak{M}$ has a factorization $G=\hat{f}_{n} \circ \hat{\alpha}_{n} \circ$ $\cdots \circ \hat{f}_{1} \circ \hat{\alpha}_{1}$, where $\hat{f}_{i}=\eta\left(f_{i}\right)$ for some $f_{i}$ in $\mathscr{K}$, and $\hat{\alpha}_{i}$ is the inverse in $\mathscr{K} / \mathfrak{M}$ for some $\eta\left(\alpha_{i}\right), \alpha_{i} \in \mathfrak{M}$.

It is immediate that

1.3. If $\mathfrak{M}$ is the class of all invertible morphisms in $\mathscr{K}$, or if $\mathfrak{M}$ is the class of all identities in $\mathscr{K}$, then $\eta: \mathscr{K} \rightarrow \mathscr{K} / \mathfrak{M}$ is an equivalence.

Proof. Since 1: $\mathscr{K} \rightarrow \mathscr{K}$ sends each $\alpha \in \mathfrak{M}$ to an invertible morphism, there is, by $\mathrm{C} 2$, a factorization $1=\Delta \circ \eta$ where $\Delta: \mathscr{K} / \mathfrak{M} \rightarrow \mathscr{K}$. This implies $\eta=\eta \circ(\Delta \circ \eta)$ $=(\eta \circ \Delta) \circ \eta$ and since the factorization through $\eta$ is unique, we must have $\eta \circ \Delta=1$. Thus $\eta: \mathscr{K} \approx \mathscr{K} / \mathfrak{M}$ is an equivalence $\left({ }^{2}\right)$.

Whenever $\mathfrak{M} \subset \mathfrak{M}^{\prime}$, the canonical projection $\eta^{\prime}: \mathscr{K} \rightarrow \mathscr{K} / \mathfrak{M}^{\prime}$ sends each $\alpha \in \mathfrak{M}$ to an invertible morphism, so there is a unique covariant functor $\Delta: \mathscr{K} / \mathfrak{M} \rightarrow \mathscr{K} / \mathfrak{M R}^{\prime}$ such that $\eta^{\prime}=\Delta \circ \eta$.

(2) The unique factorization property characterizes $(\eta, \mathscr{K} / \mathfrak{M})$ up to an equivalence; for the same technique can be used to prove: Let $\mathscr{B}$ be any category, and let $\lambda: \mathscr{K} \rightarrow \mathscr{B}$ be a covariant functor such that $\lambda(\alpha)$ is invertible for each $\alpha \in \mathfrak{M}$. Assume that for each category $\mathscr{L}$, every covariant functor $T: \mathscr{K} \rightarrow \mathscr{L}$ such that $T(\alpha)$ is invertible for each $\alpha \in \mathfrak{M}$ factors uniquely through $\lambda$. Then there is a unique equivalence $\Delta: \mathscr{K} / \mathfrak{M} \approx \mathscr{B}$ with $\lambda=\Delta \circ \eta$. 
Proposition 1.3 is a special case of

1.4. TheOREM. Let $\eta: \mathscr{K} \rightarrow \mathscr{K} / \mathfrak{M}$ be a canonical projection, and let $\overline{\mathfrak{M}}=\{f \mid \eta(f)$ is invertible $\}$. Then there exists a unique equivalence $\Delta: \mathscr{K} / \mathfrak{M} \approx \mathscr{K} / \overline{\mathfrak{M}}$ satisfying $\Delta \circ \eta=\bar{\eta}$. Moreover, $\overline{\mathfrak{M}}$ is the largest class containing $\mathfrak{M}$ for which such an equivalence holds.

Proof. Since $\mathfrak{M} \subset \overline{\mathfrak{M}}$, we have a unique $\Delta: \mathscr{K} / \mathfrak{M} \rightarrow \mathscr{K} / \overline{\mathfrak{M}}$ with $\bar{\eta}=\Delta \circ \eta$. Since $\eta(f)$ is invertible for each $f \in \overline{\mathfrak{M}}$, there is also a $\bar{\Delta}: \mathscr{K} / \overline{\mathfrak{M}} \rightarrow \mathscr{K} / \mathfrak{M}$ such that $\eta=\bar{\Delta} \circ \bar{\eta}$. Thus, $\eta=\Delta \circ \bar{\Delta} \circ \bar{\eta}$ and $\eta=\bar{\Delta} \circ \Delta \circ \eta$, so by the unique factorization property we find $\Delta \circ \bar{\Delta}=1, \bar{\Delta} \circ \Delta=1$, therefore $\Delta$ is an equivalence. To see that $\overline{\mathfrak{M}}$ is maximal, note that if $\mathfrak{M}_{0} \supset \overline{\mathfrak{M}}$, there is only one $\Delta^{\prime}$ such that $\eta_{0}=\Delta^{\prime} \circ \bar{\eta}$, and if $f \in \mathfrak{M}_{0}-\overline{\mathfrak{M}}$, then $\eta_{0}(f)$ is invertible whereas $\bar{\eta}(f)$ is not, so that $\Delta^{\prime}$ cannot be an equivalence.

2. Homotopy. Let $\mathfrak{M}$ be an arbitrary class of morphisms in an arbitrary category $\mathscr{K}$, and let $\eta: \mathscr{K} \rightarrow \mathscr{K} / \mathfrak{M}$ be the canonical projection.

2.1. Definition. Two morphisms $f, g: X \rightarrow Y$ in $\mathscr{K}$ are called $\mathfrak{M}$-homotopic (written $f \cong g \bmod \mathfrak{M}$ ) if $\eta(f)=\eta(g)$.

This has the standard properties required of a homotopy notion:

2.2. THEOREM. (a) $\mathfrak{M}$-homotopy is an equivalence relation in each $\mathscr{K}(X, Y)$.

(b) Let $f_{0}, f_{1}: X \rightarrow Y$ be $\mathfrak{M}$-homotopic. If $g: W \rightarrow X$ and $h: Y \rightarrow Z$ are any two morphisms, then $f_{0} \circ g \cong f_{1} \circ g \bmod \mathfrak{M}$ and $h \circ f_{0} \cong h \circ f_{1} \bmod \mathfrak{M}$.

(c) Each invertible morphism in $\mathscr{K}$ is an $\mathfrak{M}$-homotopy equivalence $\left({ }^{3}\right)$.

(d) If $f, g: X \rightarrow Y$ have an equalizer (or a coequalizer) $c \in \mathfrak{M}$, then $f \cong g \bmod \mathfrak{M}$.

Proof. (a)-(c) are trivial, since $\eta$ is a covariant functor. For (d): Assume, say, $c \circ f=c \circ g$; then $\eta(c) \eta(f)=\eta(c) \eta(g)$ and, since $c \in \mathfrak{M}$ so that $\eta(c)$ is invertible, we conclude that $\eta(f)=\eta(g)$.

Denote the $\mathfrak{M}$-homotopy class of $f \in \mathscr{K}(X, Y)$ by $[f]_{\mathfrak{M}}$ and the set of all $\mathfrak{M}$ homotopy classes in $\mathscr{K}(X, Y)$ by $[X, Y]_{\mathfrak{M}}$. From 2.2 it follows in the usual way that (1) there is a category $\mathscr{K}(\mathfrak{M})$ whose objects are those of $\mathscr{K}$ and whose morphisms are the $\mathfrak{M}$-homotopy classes, and (2) the function $p: \mathscr{K} \rightarrow \mathscr{K}(\mathfrak{M})$ which is the identity on objects and sends each $f$ to $[f]_{\mathfrak{M}}$, is a covariant functor $\left({ }^{4}\right)$.

This method of defining a homotopy notion in a category is reasonable, in that by a suitable choice (in fact, many choices) of $\mathfrak{M}$, one gets the usual notion of homotopy in the category Top of all topological spaces and continuous maps. This will follow from the general

$\left(^{3}\right)$ Precisely, $f: X \rightarrow Y$ is an $\mathfrak{M}$-homotopy equivalence if there exists a $g: Y \rightarrow X$ such that $f \circ g \simeq 1 \bmod \mathfrak{M}$ and $g \circ f \simeq 1 \bmod \mathfrak{M}$.

(4) In particular, if $c: A \rightarrow A$ is such that $c \circ f=c \circ g$ for every $f, g: Z \rightarrow A$, then whenever $c \in \mathfrak{M}$, the set $[Z, A]_{\mathfrak{M}}$ consists of a single element. 
2.3. THEOREM. Let $\mathscr{K}$ be a category and $\sim$ an equivalence relation in each $\mathscr{K}(A, B)$ such that the transformation $h: \mathscr{K} \rightarrow \mathscr{K}_{H}$ taking each object $A$ to itself and each $f$ to its $\sim$-class is a covariant functor.

Assume that, if $f_{0} \sim f_{1}: X \rightarrow Y$ then there exists an object $I_{X} \in \mathscr{K}$, and morphisms, $r: I_{X} \rightarrow X, i_{0}, i_{1}: X \rightarrow I_{X}, F: I_{X} \rightarrow Y$, such that $r \circ i_{j}=1$ and $f_{j}=F \circ i_{j}(j=0,1)$.

Finally, let $\mathfrak{M}$ be a class of morphisms in $\mathscr{K}$ and $\eta: \mathscr{K} \rightarrow \mathscr{K} / \mathfrak{M}$ the projection. Then

(a) If $\eta(r)$ is invertible in $\mathscr{K} / \mathfrak{M}$, then $f_{0} \sim f_{1}$ implies $f_{0} \cong f_{1} \bmod \mathfrak{M}$.

(b) If $h(\alpha)$ is invertible in $\mathscr{K}_{H}$ for each $\alpha \in \mathfrak{M}$, then $f_{0} \cong f_{1} \bmod \mathfrak{M}$ implies $f_{0} \sim f_{1}$.

Proof. (a) Assume $f_{0} \sim f_{1}$. Since $\eta(r) \circ \eta\left(i_{j}\right)=1(j=0,1)$ and $\eta(r)$ is invertible, we find that each $\eta\left(i_{j}\right)$ is invertible, and that $\eta\left(i_{0}\right)=\eta(r)^{-1}=\eta\left(i_{1}\right)$. Thus $\eta\left(f_{0}\right)=\eta(F) \eta\left(i_{0}\right)$ $=\eta(F) \eta\left(i_{1}\right)=\eta\left(f_{1}\right)$ so $f_{0} \cong f_{1} \bmod \mathfrak{M}$.

(b) Because of $\mathrm{C} 2$, the functor $h$ has a factorization $h=\Delta \circ \eta$; therefore $\eta\left(f_{0}\right)$ $=\eta\left(f_{1}\right)$ implies $h\left(f_{0}\right)=h\left(f_{1}\right)$, i.e., that $f_{0} \sim f_{1}$. This completes the proof.

Let $\mathscr{K}=$ Top and $\sim$ the usual homotopy in Top; then by taking $\mathfrak{M}$ to be any one of the four classes:

$\mathfrak{U}=$ all homotopy equivalences,

$\mathfrak{B}=$ all maps $r: X \times I \rightarrow X$, where $r(x, t)=x$,

$\mathfrak{\Im}=$ all maps $i: X \rightarrow X \times I$, where $i(x)=(x, 0)$,

$\mathfrak{X}=$ all inclusion maps $j: W \rightarrow X$, where $j(W)$ is a zero-set $\left({ }^{5}\right)$ and a strong deformation retract of $X$, it follows immediately from 2.3 that $\mathfrak{M}$-homotopy is exactly the same as the usual homotopy.

We shall say that two classes $\mathfrak{M}, \mathfrak{R}$ in a category $\mathscr{K}$ determine the same notion of homotopy, and write $[\mathfrak{M}]=[\mathfrak{N}]$, whenever $f \cong g \bmod \mathfrak{M}$ if and only if $f \cong g \bmod \mathfrak{R}$. As the examples in Top show, distinct classes may yield the same homotopy notion. We now examine briefly some questions that arise, such as: to find conditions assuring that the $\mathfrak{M}$-homotopy equivalences determine the same homotopy notion as $\mathfrak{M}$ itself.

Let $\mathfrak{M}$ be fixed, let $\eta: \mathscr{K} \rightarrow \mathscr{K} / \mathfrak{M}$ be the canonical map, and let

$$
\begin{aligned}
\overline{\mathfrak{M}} & =\{f \mid \eta(f) \text { is invertible }\} \\
E(\mathfrak{M}) & =\{f \mid \eta(f) \text { is invertible and has some } \eta(g) \text { as inverse }\} \\
& =\{f \mid f \text { is an } \mathfrak{M} \text {-homotopy equivalence }\} .
\end{aligned}
$$

It is clear that, if $\mathfrak{M} \subset \mathfrak{R}$, then $\overline{\mathfrak{M}} \subset \overline{\mathfrak{R}}$ and $E(\mathfrak{M}) \subset E(\mathfrak{R})$. The general relation of these concepts is

2.4. (a) $[\mathfrak{M}]=[\overline{\mathfrak{M}}]$ and $E(\mathfrak{M})=E(\overline{\mathfrak{M}}) \subset \overline{\mathfrak{M}}$; (b) $\overline{\mathfrak{M}}=\overline{\mathfrak{M}} \Rightarrow[\mathfrak{M}]=[\mathfrak{M}] \Rightarrow E(\mathfrak{M})$ $=E(\mathfrak{R})$.

Proof. (a) According to 1.4 , there are equivalences $\Delta: \mathscr{K} / \mathfrak{M} \approx \mathscr{K} / \overline{\mathfrak{M}}, \bar{\Delta}: \mathscr{K} / \overline{\mathfrak{M}}$ $\approx \mathscr{K} / \mathfrak{M}$ such that $\bar{\eta}=\Delta \circ \eta, \eta=\bar{\Delta} \circ \bar{\eta}$. This implies that $\eta(f)=\eta(g)$ if and only if

(5) I.e., there exists a continuous $\phi: X \rightarrow I$ with $\phi^{-1}(0)=j(W)$. 
$\bar{\eta}(f)=\bar{\eta}(g)$, so that $[\mathfrak{M}]=[\overline{\mathfrak{M}}]$. It also implies that $E(\mathfrak{M})=E(\overline{\mathfrak{M}})$; and the definition shows $E(\mathfrak{M}) \subset \overline{\mathfrak{M}}$.

(b) If $\overline{\mathfrak{M}}=\overline{\mathfrak{M}}$ then obviously $[\overline{\mathfrak{M}}]=[\overline{\mathfrak{M}}]$ and, from (a), also $[\mathfrak{M}]=[\mathfrak{R}]$. Now let $f \in E(\mathfrak{M})$; then there is a $g$ with $f \circ g \cong 1 \bmod \mathfrak{M}, g \circ f \cong 1 \bmod \mathfrak{M}$; since $[\mathfrak{M}]=[\mathfrak{R}]$, we find $f \circ g \cong 1 \bmod \mathfrak{N}, g \circ f \cong 1 \bmod \mathfrak{N}$ and so $f \in E(\mathfrak{N})$. Similarly, $f \in E(\mathfrak{N})$ implies $f \in E(\mathfrak{M})$ and the proof is complete.

In general, none of the implications in 2.4(b) are reversible.

(A) Let $\mathscr{K}$ consist of two objects $A, B$ together with the identity morphisms and a single morphism $f: A \rightarrow B$. Let $\mathfrak{M}=\left\{f, 1_{A}, 1_{B}\right\}$ and $\mathfrak{R}=\left\{1_{A}, 1_{B}\right\}$; then $[\mathfrak{M}]=[\mathfrak{N}]$ yet $\overline{\mathfrak{M}} \neq \overline{\mathfrak{N}}$ (see 1.3).

(B) Let $\mathscr{K}$ consist of three intervals $A_{i}=[0, r], r=1,2,3$, with the identity maps, the inclusion maps $j_{r s}: A_{r} \rightarrow A_{s}, r<s$, and the map $h: A_{2} \rightarrow A_{3}$ given by $h(x)=x$, $0 \leqq x \leqq 1, h(x)=1, x>1$, so that $j_{13}=j_{23} \circ j_{12}=h \circ j_{12}$. Let $\mathfrak{M}=\left\{1_{1}, 1_{2}, 1_{3}, j_{12}\right\}$ and $\mathfrak{N}=\left\{1_{1}, 1_{2}, 1_{3}\right\}$; then $[\mathfrak{M}] \neq[\mathfrak{R}]$ since $h$ is $\mathfrak{M}$-homotopic to $j_{23}$; however $E(\mathfrak{M})$ $=E(\mathfrak{N})=\mathfrak{N}$ since there is no map in $\mathscr{K}$ going backwards. Note that this shows also that $[\mathfrak{M}] \neq[\mathfrak{R}]=[E(\mathfrak{M})]$.

2.5. Definition. The class $\mathfrak{M}$ is called stable if $E(\mathfrak{M})=\overline{\mathfrak{M}}$; i.e., if the class of $\overline{\mathfrak{M}}$-homotopy equivalences is exactly $\overline{\mathfrak{M}}$.

If $\mathfrak{M}$ is stable, then $E(\mathfrak{M})$ and $\mathfrak{M}$ determine the same notion of homotopy, since $[E(\mathfrak{M})]=[\overline{\mathfrak{M}}]=[\mathfrak{M}]$. Furthermore, distinct stable classes determine distinct notions of homotopy, because, from $2.4(\mathrm{~b})$ and stability, we have

2.6. If $\mathfrak{M}, \mathfrak{R}$ are stable classes, then $\overline{\mathfrak{M}}=\overline{\mathfrak{N}} \Leftrightarrow[\mathfrak{M}]=[\mathfrak{N}] \Leftrightarrow E(\mathfrak{M})=E(\mathfrak{R})$.

The stable classes are characterized by

\subsection{TheOREM. A class $\mathfrak{M}$ in $\mathscr{K}$ is stable if and only if $\eta: \mathscr{K} \rightarrow \mathscr{K} / \mathfrak{M}$ is epic( $\left.{ }^{6}\right)$.}

Proof. "If": We need show only that $\overline{\mathfrak{M}} \subset E(\mathfrak{M})$. Let $f \in \overline{\mathfrak{M}}$; then $\eta(f)$ has an inverse $G$ in $\mathscr{K} / \mathfrak{M}$; since $\eta$ is epic, $G=\eta(g)$ for some $g$ in $\mathscr{K}$, so $f \in E(\mathfrak{M})$. "Only if": We have seen in 1.2 that each given $G$ in $\mathscr{K} / \mathfrak{M}$ has a factorization as $G=\hat{f}_{n} \circ \hat{\alpha}_{n}$ 。 $\cdots \circ \hat{f}_{1} \circ \hat{\alpha}_{1}$, where each $\hat{f}_{i}=\eta\left(f_{i}\right)$ for some $f_{i}$ in $\mathscr{K}$, and each $\hat{\alpha}_{i}$ is the inverse of some $\eta\left(\alpha_{i}\right)$. In particular, each $\alpha_{i} \in \overline{\mathfrak{M}}=E(\mathfrak{M})$, so $\eta\left(\alpha_{i}\right)$ has some $\eta\left(\beta_{i}\right)$ for inverse and, since inverses are unique, $\hat{\alpha}_{i}=\eta\left(\beta_{i}\right)$. Thus, $G=\eta\left[f_{n} \circ \beta_{n} \circ \ldots \circ f_{1} \circ \beta_{1}\right]$ and $\eta$ is epic.

2.8. Corollary. Let $\mathfrak{M}$ be any class. Then there exists a unique maximal stable class $\hat{\mathfrak{M}} \subset \mathfrak{M}$ with the following property: if $\mathfrak{N} \subset \mathfrak{M}$ is stable, then $\mathfrak{R} \subset \hat{\mathfrak{M}}$.

Proof. In view of our constructions, the empty class $\varnothing \subset \mathfrak{M}$ is (1.3) clearly stable. It is therefore enough to show that the union of any family of stable classes is stable. Let $\left\{\mathfrak{M}_{\beta} \mid \beta \in B\right\}$ be the family of all stable classes in $\mathfrak{M}$, so that each $\eta_{\beta}: \mathscr{K} \rightarrow \mathscr{K} / \mathfrak{M}_{\beta}$ is epic; letting $\hat{\mathfrak{R}}=\bigcup \mathfrak{M}_{\beta}$, we will prove that $\eta: \mathscr{K} \rightarrow \mathscr{K} / \hat{\mathfrak{M}}$ is also epic. Each morphism $G$ in $\mathscr{K} / \hat{\mathfrak{M}}$ has a factorization $G=\hat{f}_{n} \circ \hat{\alpha}_{n} \circ \ldots \circ \hat{f}_{1} \circ \hat{\alpha}_{1}$,

$\left.{ }^{(}\right)$I.e., for each $A, B$ and $f \in$ hom $(A, B)$, there exists an $f: A \rightarrow B$ with $\eta(f)=f$. Note that the stability of $\mathfrak{M}$ implies that each hom $(A, B)$ is in fact a set, cf. footnote 14,15 . 
where each $\hat{f}_{i}=\eta\left(f_{i}\right)$ for some $f_{i}$ in $\mathscr{K}$. Each $\alpha_{i}$ lies in some $\mathfrak{M}_{i}$ so there is a $\beta_{i}$ in $\mathscr{K}$ with $\eta\left(\beta_{i}\right)=\alpha_{i}$ in $\mathscr{K} / \mathfrak{M}_{i}$. Since (1.4) there is an $\hat{\eta}_{i}: \mathscr{K} / \mathfrak{M}_{i} \rightarrow \mathscr{K} / \hat{\mathfrak{M}}$ with $\eta=\hat{\eta}_{i} \circ \eta_{i}$, it follows that $\eta\left(\beta_{i}\right)=\hat{\alpha}_{i}$ in $\mathscr{K} / \hat{\mathfrak{M}}$ and therefore that $\eta\left(f_{n} \circ \beta_{n} \circ \ldots \circ f_{1} \circ \beta_{1}\right)=G$. This completes the proof.

The maximal stable class $\hat{\mathfrak{M}}$ may not give the same notion of homotopy as $\mathfrak{M}$. In fact, there are notions of $\mathfrak{M}$-homotopy that cannot be obtained from any stable class: in example (B) above, $\mathfrak{M}=\mathfrak{N}$ and, indeed, $\mathfrak{M}$-homotopy cannot be obtained from a stable class. Moreover, $E(\mathfrak{M}) \neq E(\hat{\mathfrak{M}})$ in general (cf. Appendix A5).

The machinery developed so far can be applied to any covariant functor $\Phi: \mathscr{K} \rightarrow \mathscr{L}$ to introduce into $\mathscr{K}$ a notion of homotopy induced (or adapted to) the functor $\Phi$.

2.9. Definition. Let $\Phi: \mathscr{K} \rightarrow \mathscr{L}$ be any covariant functor. Let

$$
\mathfrak{M}(\Phi)=\{f \text { in } \mathscr{K} \mid \Phi(f) \text { is invertible }\} .
$$

The homotopy in $\mathscr{K}$ determined by the class $\mathfrak{M}(\Phi)$ is called $\Phi$-homotopy.

The category $\mathscr{K} / \mathfrak{M}(\Phi)$ is written $\mathscr{K}^{\Phi}$ and is essentially the category first studied by Bauer in [1]. Observe that, because of $\mathrm{C} 2$, the functor $\Phi$ has a unique factorization $\Phi=\Delta \circ \eta$ through $\mathscr{K}^{\Phi}$. It follows from this that $\mathfrak{M}(\Phi)=[\mathfrak{M}(\Phi)]^{-}$always : for, if $f \in[\mathfrak{M}(\Phi)]^{-}$, then $\eta(f)$ is invertible, therefore so also is $\Delta(\eta(f))=\Phi(f)$, consequently $f \in \mathfrak{M}(\Phi)$; thus, $[\mathfrak{M}(\Phi)]^{-} \subset \mathfrak{M}(\Phi)$ and the opposite inclusion is trivial.

We give some applications of $\Phi$-homotopy.

(1) Let $\Phi:$ Top $\rightarrow$ Ens be the forgetful functor. Then $f$ is $\Phi$-homotopic to $g$ if and only if $f=g$. For, consider the factorization $\Phi=\Delta \circ \eta$ through $\mathscr{K}^{\Phi}$. If $\eta(f)$ $=\eta(g)$, then $\Phi(f)=\Phi(g)$ so that $f=g$ as maps of sets; and the converse is trivially true. The class $\mathfrak{M}=\mathfrak{M}(\Phi)$ consists of all bijective continuous maps, whereas $E(\mathfrak{M})$ is the class of all bijective bicontinuous maps (i.e., homeomorphisms).

(2) Let $\mathscr{K}$ be the category of spaces dominated by CW-complexes, let $\mathscr{G}^{z}$ be the category of graded groups and degree zero homomorphisms, and let $\pi: \mathscr{K} \rightarrow \mathscr{G}^{z}$ be the total homotopy functor. Then two maps are $\pi$-homotopic if and only if they are homotopic. For, we apply 2.3 because (a) each projection $r: X \times I \rightarrow X$ is such that $\pi(r)$ is invertible, therefore $r \in \mathfrak{M}(\pi)$, consequently $\eta(r)$ is invertible, and (b) if $f \in \mathfrak{M}(\pi)$, i.e., if $\pi(f)$ is invertible, then, by Whitehead's theorem [6] $f$ is a homotopy equivalence and therefore $h(f)$ is invertible. Note that, again using Whitehead's theorem, the class $\mathfrak{M}(\pi)$ is stable, so that $\eta: \mathscr{K} \rightarrow \mathscr{K}^{\pi}$ is epic. Similarly, if $\mathscr{K}$ is the category of Kan-complexes, then $\pi$-homotopy in $\mathscr{K}$ is exactly Kan-homotopy.

(3) Let $\mathscr{K}$ be the category of all simply connected CW-complexes, and $H: \mathscr{K} \rightarrow \mathscr{G}^{z}$ the total homology functor. Exactly the same considerations as in (2) reveal $H$-homotopy to be the usual homotopy. Using $H: \mathscr{K} \rightarrow \mathscr{G}^{z}$ on the category of css complexes yields a homotopy notion extending the usual one for Kancomplexes. And on the category $\mathscr{K}$ of chain-complexes, $H$-homotopy yields the usual notion of chain-homotopy.

Thus, the various notions of homotopy encountered, and usually defined 
differently, in various categories turn out to be essentially the homotopy relation determined in the above manner by a suitable covariant functor. For arbitrary contravariant functors, one turns to the dual category.

3. Fibrations. Each class $\mathfrak{M}$ of morphisms in a category $\mathscr{K}$ determines a concept of fibration in $\mathscr{K}$ :

3.1. Definition. A morphism $p: E \rightarrow B$ in $\mathscr{K}$ is called an $\mathfrak{M}$-fibration if for each diagram

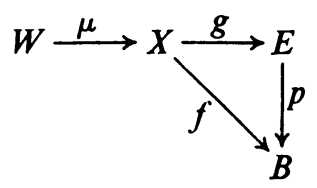

in which $\mu \in \mathfrak{R}$ and $p \circ g \circ \mu=f \circ \mu$, there exists a $g^{\prime}: X \rightarrow E$ in $\mathscr{K}$ with $g \circ \mu$ $=g^{\prime} \circ \mu$ and $p \circ g^{\prime}=f$.

We are requiring simply that every triangle involving $p$ which can be equalized by a morphism belonging to $\mathfrak{M}$, can be made itself commutative. Note that if $\mathfrak{M}$-homotopy is used in $\mathscr{K}$, the conditions $p \circ g \circ \mu=f \circ \mu$ and $g \circ \mu=g^{\prime} \circ \mu$ imply, by $2.2(\mathrm{~d})$, that $p \circ g \cong f \bmod \mathfrak{M}$ and $g \cong g^{\prime} \bmod \mathfrak{M}$. The relations between $\mathfrak{M}$ homotopy and $\mathfrak{M}$-fibration will be considered after we verify that the class of $\mathfrak{M}$-fibrations has the properties usually required of fibrations.

3.2. TheOREM. Let $\mathfrak{M}$ be a fixed class of morphisms in $\mathscr{K}$. Then:

(a) If $p: E \rightarrow B$ is invertible, then $p$ is an M-fibration.

(b) If $p_{1}: E_{1} \rightarrow E_{0}$ and $p_{0}: E_{0} \rightarrow B$ are $\mathfrak{M}$-fibrations, so also is $p_{0} \circ p_{1}: E_{1} \rightarrow B$.

(c) If $p: E \rightarrow B$ is an $\mathfrak{M}$-fibration, and if

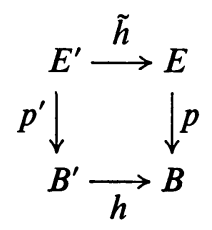

is a cartesian diagram $\left({ }^{7}\right)$, then $p^{\prime}$ is also an $\mathfrak{M}$-fibration; and $h$ can be lifted into $E$ if and only if $p^{\prime}$ has a section $\left({ }^{8}\right)$.

Proof. (a) is trivial.

(7) In the terminology of Mitchell [4] the indicated diagram would be called a pullback of $p, h$.

(8) I.e., an $s: B^{\prime} \rightarrow E^{\prime}$ such that $p^{\prime} \circ s^{\prime}=1$. 
(b) Given

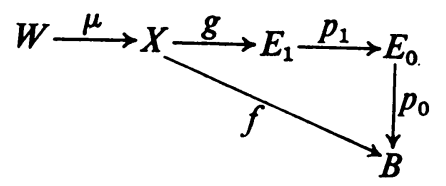

with $p_{0} \circ p_{1} \circ g \circ \mu=f \circ \mu$ and $\mu \in \mathfrak{M}$, there exists first a $G: X \rightarrow E_{0}$ with $p_{0} \circ G=f$, $G \circ \mu=p_{1} \circ g \circ \mu$; and then a $g^{\prime}: X \rightarrow E_{1}$ with $p_{1} \circ g^{\prime}=G, g^{\prime} \circ \mu=g \circ \mu$; since $p_{0} \circ p_{1} \circ g=p_{0} \circ G=f$, the required morphism is $g^{\prime}$.

(c) We are given

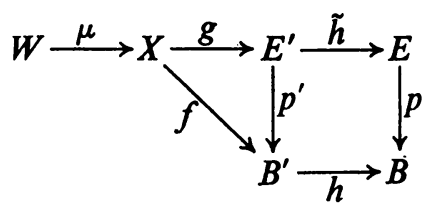

where the square is cartesian and $f \circ \mu=p^{\prime} \circ g \circ \mu$. Thus, $h \circ f \circ \mu=p \circ \tilde{h} \circ g \circ \mu$ so, because $p$ is an $\mathfrak{M}$-fibration, there is a $G: X \rightarrow E$ such that $G \circ \mu=\tilde{h} \circ g \circ \mu$ and $p \circ G=h \circ f$. Because the square is cartesian and $p \circ G=h \circ f$, there exists a unique $g^{\prime}: X \rightarrow E^{\prime}$ such that $\tilde{h} \circ g^{\prime}=G$ and $p^{\prime} \circ g^{\prime}=f$. Since also $\tilde{h} \circ\left(g^{\prime} \circ \mu\right)=G \circ \mu=\tilde{h}$ $\circ(g \circ \mu)$ and $p^{\prime} \circ(g \circ \mu)=f \circ \mu=p^{\prime} \circ\left(g^{\prime} \circ \mu\right)$, the uniqueness of a morphism $W \rightarrow E^{\prime}$ satisfying these two conditions in a cartesian diagram shows $g^{\prime} \circ \mu=g \circ \mu$ and therefore we find that $p^{\prime}$ is an $\mathfrak{M}$-fibration. The second part is proved in a similar manner.

We now examine the notion of $\mathfrak{M}$-fibration in Top, using the classes, and the symbols for those classes, that are listed following Theorem 2.3. We have

3.3 ThEOREM. Let $\mathfrak{M}=\Im$ in Top. Then $\Im$-homotopy is the classical notion, and $p: E \rightarrow B$ is $a$ ঔ-fibration if and only if it is a Hurewicz fibration.

Proof. The first part was established in 2.3. Let now $p: E \rightarrow B$ be a $\Im$-fibration; we show it has the covering homotopy property, i.e., that it is a Hurewicz fibration. We start with the commutative diagram

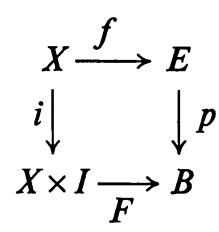

where $i(x)=(x, 0)$. Let $r: X \times I \rightarrow X$ be the map $r(x, t)=x$; we then have

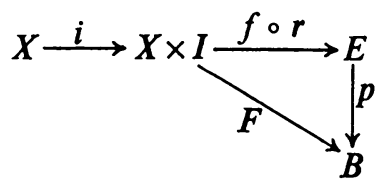


where $F \circ i=p \circ f=p \circ f \circ r \circ i$. Since $p$ is a $\Im$-fibration, there is an $\bar{F}: X \times I \rightarrow E$ with $p \circ \bar{F}=F$ and $\bar{F} \circ i=f \circ r \circ i=f$, so that $\bar{F}$ is a homotopy of $f$ covering $F$, and therefore $p$ is a Hurewicz fibration.

Conversely, let $p: E \rightarrow B$ be a Hurewicz fibration. The morphisms in $\Im$ being simply the maps $i: X \rightarrow X \times I$, we consider any diagram

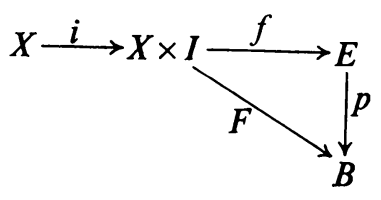

in which $p \circ f \circ i=F \circ i$; since $p$ is a Hurewicz fibration, there is a homotopy $\bar{F}$ of $f \circ i$ covering $F$, so that $p \circ \bar{F}=F$ and $f \circ i=\bar{F} \circ i$. Thus, $p$ is a $\mathfrak{W}$-fibration, and the proof is complete.

If $\mathfrak{M} \subset \mathfrak{N}$, it is $\operatorname{clear}\left({ }^{9}\right)$ that $\{\mathfrak{N}$-fibrations $\} \subset\{\mathfrak{M}$-fibrations $\}$. Moreover,

(a) Two classes may determine the same notion of homotopy, but distinct notions of fibration.

Example in Top: We have seen that $\mathfrak{B}$ determines the same homotopy notion as $\Im$, that is, the usual notion of homotopy. However, every continuous map is a $\mathfrak{B}$-fibration: for, clearly, $\mathfrak{B} \subset$ (all continuous surjections), and, if $\mu$ is surjective, the condition $p \circ g \circ \mu=f \circ \mu$ of Definition 3.1 implies that $p \circ g=f$; thus, every continuous $p: E \rightarrow B$ belongs to \{surjective map fibrations $\} \subset\{\mathfrak{B}$-fibrations\}.

(b) Two classes may determine the same notion of fibration but distinct notions of homotopy.

Example in Top: Let $\mathfrak{M}=$ class of all identity maps $1_{A}: A \rightarrow A$. Then every continuous map is an $\mathfrak{M}$ - and also a $\mathfrak{B}$-fibration; yet $\mathfrak{B}$-homotopy is the usual notion of homotopy, whereas we have seen that $\mathfrak{M}$-homotopy is simply equality.

From this viewpoint, the notions of homotopy and of fibration are independent. In the category Top, the Hurewicz fibrations appear as a concept dependent on a particular class of morphisms that happens to yield the usual homotopy notion, rather than as a concept dependent on the homotopy notion itself $\left({ }^{10}\right)$.

Returning to 3.1, we shall establish a simple criterion for two classes to determine the same notion of fibration. This is based on

3.4. THEOREM. Let $\mathfrak{M}, \mathfrak{Q}$, be two classes of morphisms in $\mathscr{K}$. Assume that for each $\mu: W \rightarrow X, \mu \in \mathfrak{M}$, there exist $a \lambda: Y \rightarrow Z, \lambda \in \mathfrak{L}$ and morphisms in $\mathscr{K}$ such that

$\left({ }^{9}\right)$ We denote the class of $\mathfrak{M}$-fibrations by $\{\mathfrak{M}$-fibrations $\}$.

$\left.{ }^{(10}\right)$ The class $\mathscr{H}$ of Hurewicz fibrations does not determine the usual homotopy: in fact, since every constant map $f: E \rightarrow e$ is a Hurewicz fibration, it follows (cf. footnote 4) that for each $X, Y$, all $f, g: X \rightarrow Y$ are $\mathscr{H}$-homotopic. 


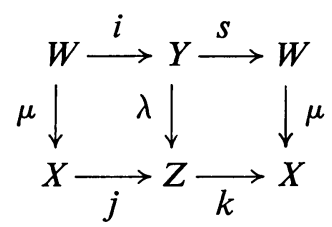

is commutative and $k \circ j=1$. Then $\{\mathfrak{Q}$-fibrations $\} \subset\{\mathfrak{M}$-fibrations $\}$.

Proof. Let $p: E \rightarrow B$ be an \&-fibration. Given a diagram

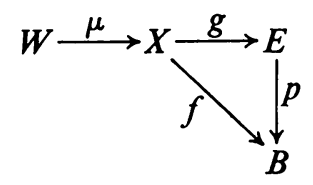

with $p \circ g \circ \mu=f \circ \mu$ and $\mu \in \mathfrak{M}$, we find that $p \circ g \circ k \circ \lambda=p \circ g \circ \mu \circ s=f \circ \mu \circ s$ $=f \circ k \circ \lambda$ so, since $p$ is an $\mathbb{L}$-fibration, there is a $\Gamma: Z \rightarrow E$ with $p \circ \Gamma=f \circ k$ and $\Gamma \circ \lambda=g \circ k \circ \lambda$. Letting $G=\Gamma \circ j$, we find $p \circ G=p \circ \Gamma \circ j=f \circ k \circ j=f$, and $G \circ \mu=\Gamma \circ j \circ \mu=g \circ k \circ \lambda \circ i=g \circ k \circ j \circ \mu=g \circ \mu$, so that $p$ is an $\mathfrak{M}$-fibration.

As an application,

3.5. In Top, $\{\mathfrak{X}$-fibrations $\}=\{$ Hurewicz fibrations $\}$.

Proof. Clearly, $\Im \subset \mathfrak{X}$, so $\{\mathfrak{X}$-fibrations $\} \subset\{\Im$-fibrations $\}$. For the converse, let $\mu: W \rightarrow X, \mu \in \mathfrak{X}$, be given; we identify $W$ with a subset of $X$ in order to cut down excessive notation, and let $\Phi: r \cong 1$ be a strong deformation retraction. Choose $\phi: X \rightarrow I$ vanishing exactly on $W$, and define $\bar{\Phi}: X \times I \rightarrow X$ by

$$
\begin{aligned}
\bar{\Phi}(x, t) & =\Phi(x, t / \phi(x)), & & x \notin W, \\
& =\Phi(x, 1), & & x \in W,
\end{aligned}
$$

which is easily verified to be continuous. Then the diagram

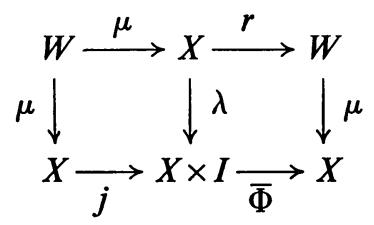

where $r=$ retraction, $\mu=$ inclusion, $\lambda(x)=(x, 0)$ and $j(x)=(x, \phi(x))$ is easily seen to be commutative. Thus, $\{\Im$-fibrations $\} \subset\{\mathfrak{X}$-fibrations $\}$ and, with 3.3 , the theorem is proved.

We recall that, in Top, a fibration is called regular whenever a covering homotopy can always be chosen stationary at all the points where the given homotopy is stationary $\left({ }^{11}\right)$. Let $\tilde{X}$ be the family of all inclusions $\mu: A \rightarrow X$, where $A$ is a

(11) An example of a nonregular Hurewicz fibration, as well as a general condition on a topological space $B$ that assures the regularity of every Hurewicz fibration over $B$, is given in [5]. 
strong deformation retract of $X$, and let $\tilde{\mathfrak{S}}$ be the class of all maps $\lambda_{B}: X \rightarrow X_{B}=$ $(X \times I) \bigcup_{r \mid B \times I} B, r \in \mathfrak{B}$, where $B \subset X$ is arbitrary, and $\lambda_{B}=\rho_{B} \circ i$ with $i \in \mathfrak{\Im}$ and $\rho_{B}: X \times I \rightarrow X_{B}$ the identification. Clearly, $\mathfrak{X} \subset \tilde{\mathfrak{X}}$ and $\mathfrak{\Im} \subset \tilde{\mathfrak{B}} \subset \mathfrak{X}$, so that every $\tilde{\mathfrak{X}}$ and every $\tilde{\mathfrak{W}}$-fibration is a Hurewicz fibration.

3.7. THEOREM $\left({ }^{12}\right)$. In Top $\{\mathfrak{X}-$ fibrations $\}=\{\tilde{\mathfrak{G}}-$ fibrations $\}=\{$ regular Hurewicz fibrations\}.

Proof. The proof that $\{\tilde{\mathfrak{S}}$-fibrations $\}=$ \{regular Hurewicz fibrations $\}$ is a repetition of that for 3.3, with $X \times I$ replaced by suitable $X_{B}$ and $\mathfrak{\Im}$ replaced by $\tilde{\mathfrak{Y}}$. Because $\tilde{\mathfrak{W}} \subset \tilde{\mathfrak{X}}$, we have $\{\tilde{\mathfrak{X}}$-fibrations $\} \subset\{\tilde{\mathfrak{W}}$-fibrations $\}$. To prove the converse inclusion, let $\mu: A \rightarrow X, \mu \in \tilde{\mathfrak{X}}$, be given, let $\Phi: r \cong 1$, be a strong deformation retraction of $X$ onto $A$; then a continuous $\bar{\Phi}: X_{A} \rightarrow X$ such that $\bar{\Phi} \circ \rho_{A}=\Phi$ exists. Let $i_{1}: X \rightarrow X \times I$ be the map $x \rightarrow(x, 1)$; then the diagram

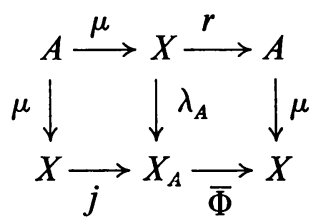

with $j=\rho_{A} \circ i_{1}$, is commutative and $\bar{\Phi} \circ j=1$. Thus, by 3.5 , the proof is complete.

As in the previous section, a covariant functor $\Phi: \mathscr{K} \rightarrow \mathscr{L}$ gives rise to a notion of $\Phi$-fibration, i.e., $\mathfrak{M}(\Phi)$-fibration. Observe that, if $\pi: \mathscr{K} \rightarrow \mathscr{G}^{z}$ is the total homotopy functor on the category of spaces dominated by $\mathrm{CW}$-complexes, then the corresponding notion of $\Phi$-fibration is not that of Hurewicz fibration, but of a certain subset: for, $\mathfrak{M}(\Phi)=$ set of all homotopy equivalences and $\mathfrak{} \subset \mathfrak{M}(\Phi)$ so that $\{\mathfrak{M}(\Phi)$-fibrations $\} \subset\{\Im$-fibrations $\}=\{$ Hurewicz fibrations $\}$.

\section{Cofibrations.}

4.1. Definition. Let $\mathfrak{M}$ be a class of morphisms in a category $\mathscr{K}$. A morphism $j: B \rightarrow E$ in $\mathscr{K}$ is called an $\mathfrak{M}$-cofibration if for each diagram

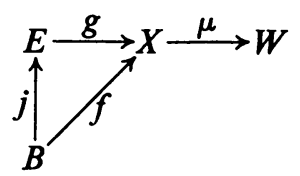

in which $\mu \in \mathfrak{M}$ and $\mu \circ g \circ j=\mu \circ f$, there exists a $g^{\prime}: E \rightarrow X$ with $g^{\prime} \circ j=f$ and $\mu \circ g^{\prime}=\mu \circ g$.

This concept depends on the class $\mathfrak{M}$ itself rather than on the homotopy notion that $\mathfrak{M}$ determines. In fact, the corresponding homotopy notions may be the same, and the associated cofibrations different, since

4.2. THEOREM. In the category Top,

${ }^{(2)}$ For $\tilde{\mathfrak{x}}$-fibrations, this theorem was suggested by the referee. 
(a) $\{\Im$-cofibrations $\}=\{\mathfrak{X}$-cofibrations $\}=$ all morphisms in Top;

(b) $\{\mathfrak{B}$-cofibrations $\}=$ class of all $j: B \rightarrow E$ such that every continuous $\phi: B \rightarrow I$ has a factorization $\phi=\psi \circ j$ where $\psi: E \rightarrow I$.

Proof. (a) is trivial. (b) Assume that $j: B \rightarrow E$ is a $\mathfrak{B}$-cofibration. Let $\phi: B \rightarrow I$ be given and consider

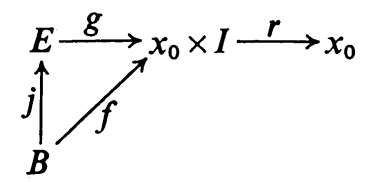

where $f(b)=\left(x_{0}, \phi(b)\right)$ and $g(e)=\left(x_{0}, 0\right)$. By hypothesis, there exists a $g^{\prime}: E \rightarrow x_{0} \times I$ such that $r \circ g^{\prime}=r \circ g$ and $g^{\prime} \circ j=f$, i.e., $g^{\prime}(e)=\left(x_{0}, \psi(e)\right)$ and $\psi \circ j=\phi$.

Conversely, assume that the property is satisfied for $j: B \rightarrow E$, and consider any diagram

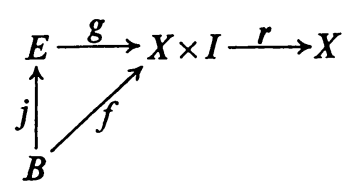

where $r \circ f=r \circ g \circ j$. Using the projections on each factor, write $f(b)=\left(f_{X}(b), f_{I}(b)\right)$ and $g(e)=\left(g_{X}(e), g_{I}(e)\right)$; the commutativity requirement assures $f_{X}(b)=g_{X}(j b)$; but $f_{I}(b)=\psi j(b)$ for suitable $\psi: E \rightarrow I$, so that $g^{\prime}(e)=\left(g_{X}(e), \psi(e)\right)$ is the desired map.

In particular, if $j: B \rightarrow E$ is inclusion onto a retract of $E$, then $j$ is a $\mathfrak{B}$-cofibration. Moreover,

4.3. If $B$ is a functional Hausdorff space $\left({ }^{13}\right)$, then each $\mathfrak{B}$-cofibration $j: B \rightarrow E$ is injective.

Proof. Let $j: B \rightarrow E$ be a map such that $j\left(b_{0}\right)=j\left(b_{1}\right)$ for some $b_{0} \neq b_{1}$. There exists a map $\phi: B \rightarrow I$ with $\phi\left(b_{0}\right)=0, \phi\left(b_{1}\right)=1$; and $\phi$ cannot factor as $\phi=\psi \circ j$.

It is interesting to note that, within this framework, $\{\Im$-fibrations $\} \equiv\{$ Hurewicz fibrations $\}$ has $\{\mathfrak{\Im}$-cofibrations $\} \equiv\{$ all morphisms $\}$ for dual; and that $\{\mathfrak{B}$-fibrations $\}$ $\equiv\{$ all morphisms $\}$ has $\{\mathfrak{B}$-cofibrations $\} \equiv$ (a restricted class of morphisms) for dual.

For a covariant functor $\Phi: \mathscr{K} \rightarrow \mathscr{L}$ the notion of $\Phi$-cofibration is defined, in the customary manner, to be that of $\mathfrak{M}(\Phi)$-cofibration. For contravariant functors, one uses the dual category, in which fibrations are exchanged against cofibrations.

5. Weak $\mathfrak{M}$-fibrations. We recall that, in the category Top, a map $p: E \rightarrow B$ is called a Dold fibration (=a map with the WCHP in [2]) if for each commutative diagram

$\left.{ }^{(13}\right)$ I.e., for each pair $b_{0} \neq b_{1}$ of points in $B$, there exists a continuous $\phi: B \rightarrow I$ such that $\phi\left(b_{0}\right)=0$ and $\phi\left(b_{1}\right)=1$. 


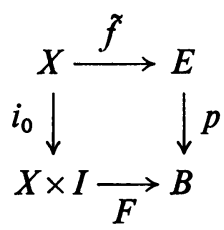

there exists a map $\tilde{F}: X \times I \rightarrow E$ such that $p \circ \tilde{F}=F$ and $\tilde{F} \circ i_{0}$ is fiber-homotopic to $\tilde{f}$.

For any $X \in$ Top, let $\rho=\rho_{X}: X \times I \rightarrow X \times I$ denote the map

$$
\begin{aligned}
\rho(x, t) & =(x, 0), & & 0 \leqq t \leqq \frac{1}{2}, \\
& =(x, 2 t-1), & & \frac{1}{2} \leqq t \leqq 1,
\end{aligned}
$$

and let $\Re=\left\{\rho_{X} \mid X \in\right.$ Top $\}$. Then

5.1. Lemma. A map $p: E \rightarrow B$ is a Dold fibration if and only if for each diagram

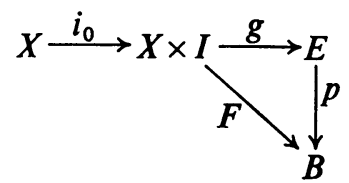

with $p \circ g \circ i_{0}=F \circ i_{0}$, there exists a $g^{\prime}: X \times I \rightarrow E$ such that $g \circ i_{0}=g^{\prime} \circ i_{0}$ and $p \circ g^{\prime}$ $=F \circ \rho$.

Because the proof of 5.1 is entirely analogous to that of 3.3, it is omitted.

Let $\mathscr{K}$ be an arbitrary category and $\mathfrak{M}$ be any family of its morphisms. With 5.1 in mind, we make the following

5.2. Definition. A morphism $p: E \rightarrow B$ in $\mathscr{K}$ is called a weak $\mathfrak{M}$-fibration if for each diagram

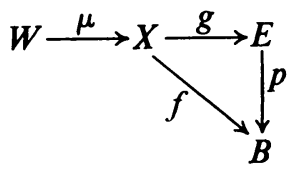

in which $\mu \in \mathfrak{M}$ and $p \circ g \circ \mu=f \circ \mu$, there exists a $g^{\prime}: X \rightarrow E$ in $\mathscr{K}$, and an $r: X \rightarrow X$ in $\mathfrak{M}$ such that $r \circ \mu=\mu, g \circ \mu=g^{\prime} \circ \mu$, and $p \circ g^{\prime}=f \circ r$.

REMARKS. (1) If $\mathfrak{M}$ is such that $\mathfrak{M} \cap \mathscr{K}(X, X)=\left\{1_{X}: X \rightarrow X\right\}$ for each $X \in \mathscr{K}$, then $\{$ weak $\mathfrak{M}$-fibrations $\}=\{\mathfrak{M}$-fibrations $\}$.

(2) If $\mathfrak{M}$ is any class that contains all the identity morphisms, then each $\mathfrak{M}$ fibration is also a weak $\mathfrak{M}$-fibration.

(3) Unlike the $\mathfrak{M}$-fibrations, it is not in general true that $(\mathfrak{M} \subset \mathfrak{R}) \Rightarrow(\{$ weak $\mathfrak{N}$ fibrations $\} \subset\{$ weak $\mathfrak{M}$-fibrations $\})$.

5.3. Definition. For any family $\mathfrak{M}$ of morphisms in Top, we let $\mathfrak{M}_{e}=\mathfrak{M} \cup$ \{all identities\} and $\mathfrak{M}^{*}=\mathfrak{M}_{e} \cup \mathfrak{R}$. Then

5.4. TheOREM. In the category Top, 
(a) $\left\{\right.$ weak $\mathfrak{X}_{e}$-fibrations $\}=\left\{\right.$ weak $\mathfrak{\Im}_{e}$-fibrations $\}=\{$ Hurewicz fibrations $\}$;

(b) $\left\{\right.$ weak $\mathfrak{X}^{*}$-fibrations $\}=\left\{\right.$ weak $\Im^{*}$-fibrations $\}=\{$ Dold fibrations $\}$.

Proof. (a) follows immediately from Remark (1) above and Theorems 3.3, 3.5. To prove (b): Observe that we have

$$
\left\{\text { weak } \mathfrak{X}^{*} \text {-fibrations }\right\} \subset\left\{\text { weak } \mathfrak{\Im}^{*} \text {-fibrations }\right\}
$$

because $\Im \subset \mathfrak{X}$ and all morphisms $r$ that can possibly appear in Definition 5.2 are in $\Re_{e}$. The converse inclusion follows by an argument entirely analogous to that in the proof of 3.5. Finally, $\left\{\right.$ weak $\Im^{*}$-fibrations $\}=\{$ Dold fibrations $\}$ by 5.1 . This completes the proof (b).

Everything which has been said for weak $\mathfrak{M}$-fibrations can be dualized in the usual fashion, to yield weak $\mathfrak{M}$-cofibrations.

Appendix. In this section, we will give a proof of Theorem 1.1. We also include an example to show that in general $E(E(\mathfrak{M})) \neq E(\mathfrak{M})$ and $E(\mathfrak{M}) \neq E(\hat{\mathfrak{M}})$.

Let $\mathscr{K}$ be an arbitrary category, and let $\mathfrak{M}$ be any subclass of its morphisms; the case that $\mathfrak{M}$ is the family of all morphisms in $\mathscr{K}$ is not excluded. We denote the elements of $\mathfrak{M}$ by small Greek letters, $\alpha, \beta, \ldots$

Given $A, B \in$ ob $(\mathscr{K})$, by an $\mathfrak{M}$-word $m=\left(A, X_{1}, \ldots, X_{2 n-1}, B\right)$ from $A$ to $B$ we mean a finite chain of morphisms and objects

$$
A \stackrel{\alpha_{1}}{\longleftarrow} X_{1} \stackrel{f_{1}}{\longrightarrow} X_{2} \stackrel{\alpha_{2}}{\longleftarrow} X_{3} \stackrel{f_{2}}{\longrightarrow} \cdots \stackrel{\alpha_{n}}{\longleftarrow} X_{2 n-1} \stackrel{f_{n}}{\longrightarrow} B
$$

in which the $\leftarrow$ and $\rightarrow$ alternate, and each morphism $\alpha_{i}$ "in the wrong direction" either belongs to $\mathfrak{M}$ or is an identity morphism. The class of all $\mathfrak{M}$-words from $A$ to $B$ is denoted by $\mathfrak{M}(A, B)$.

We introduce an equivalence relation $\sim$ in $\mathfrak{M}(A, B)$ by

A.1. Definition. Let $m, \hat{m} \in \mathfrak{M}(A, B)$. Declare $m \sim \hat{m}$ if there is a finite sequence $m=m_{1}, m_{2}, \ldots, m_{s}=\hat{m}$ of elements of $\mathfrak{M}(A, B)$ in which each $m_{i}$ is obtained from $m_{i-1}$ (or from $m_{i+1}$ ) by one of the following two operations:

(i) A pushout operation: replacement of a segment

$$
\stackrel{f}{\longrightarrow} X \stackrel{\alpha}{\longleftarrow} Y \stackrel{g}{\longrightarrow} Z \stackrel{\beta}{\longleftarrow} .
$$

in $m_{i-1}\left(\right.$ or $\left.m_{i+1}\right)$ by

$$
\stackrel{u \circ f}{\longleftarrow} W \stackrel{v \circ \beta}{\longrightarrow}
$$

if there is a commutative diagram

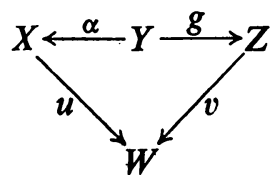


and $v \circ \beta \in \mathfrak{M}$ or is an identity morphism. This operation is denoted by

$$
P O(X, Y, Z: u, W, v) \text {. }
$$

(ii) A pullback operation: replacement of a segment

$$
\stackrel{\alpha}{\longleftarrow} X \stackrel{f}{\longrightarrow} Y \stackrel{\beta}{\longleftarrow} Z \stackrel{g}{\longrightarrow} \text {. }
$$

in $m_{i-1}\left(\right.$ or $\left.m_{i+1}\right)$ by

$$
\stackrel{\alpha \circ u}{\longleftarrow} W \stackrel{g \circ v}{\longrightarrow}
$$

if there is a commutative diagram

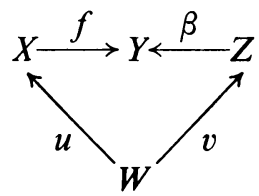

and $\alpha \circ u \in \mathfrak{M}$ or is an identity morphism. This operation is denoted by

$$
P B(X, Y, Z ; u, W, v) .
$$

It is clear that $\sim$ is an equivalence relation in $\mathfrak{M}(A, B)$; the equivalence class of $m$ is denoted by $[m]$. Furthermore, the map $\mathfrak{M}(A, B) \otimes \mathfrak{M}(B, C) \rightarrow \mathfrak{M}(A, C)$ defined by

$$
\left(A, X_{1}, \ldots, X_{2 n-1}, B\right) \otimes\left(B, Y_{1}, \ldots, C\right)=\left(A, X_{1}, \ldots, X_{2 n-1}, B, Y_{1}, \ldots, C\right)
$$

is associative, and the equivalence class of $m \otimes m^{\prime}$ depends only on that of $m$ and of $m^{\prime}$.

A.2. Definition. The category $\mathscr{K} / \mathfrak{M}$ is that having for objects: $\{A \mid A \in \operatorname{ob}(\mathscr{K})\}$; for morphisms hom $(A, B)$ : the equivalence classes $\left({ }^{14}\right)$ in $\mathfrak{M}(A, B)$, with the composition law hom $(B, C) \circ$ hom $(A, B) \rightarrow$ hom $(A, C)$ given by $\left[m^{\prime}\right] \circ[m]=\left[m \otimes m^{\prime}\right]$.

The identity $\left[e_{A}\right] \in \operatorname{hom}(A, A)$ is the morphism $[A \stackrel{1}{\leftarrow} A \stackrel{1}{\rightarrow} A]$ : for, given $[m] \in \operatorname{hom}(B, A)$ we find that $m \otimes e_{A}$ is a word of form

$$
\cdots \stackrel{f}{\longrightarrow} A \stackrel{1}{\longleftarrow} A \stackrel{1}{\longrightarrow} A
$$

and $P B(X, A, A ; 1, X, f)$ shows $m \otimes e_{A} \sim m$; similarly, $e_{A} \otimes m^{\prime} \sim m^{\prime}$ for $m^{\prime} \in \mathfrak{M}(A, B)$ so $\left[e_{A}\right]$ is the (unique) identity morphism of $A$.

A.3. Let each morphism in a word $m \in \mathfrak{M}(A, B)$ be either a member of $\mathfrak{M}$ or an identity. Then $[m]$ is invertible in $\mathscr{K} / \mathfrak{M}$; and its inverse is represented by $m$ written in reverse order.

$\left.{ }^{(14}\right)$ If $\mathscr{K}$ is not a small category, hom $(A, B)$ need not be a set. However, in all the choices of $\mathscr{K}$ and $\mathfrak{M}$ considered in this paper, the class hom $(A, B)$ can be proved to be a set, so that the construction of this category is legitimate. 
Proof. Letting $m^{\prime}$ be the word in reverse order, we have $m^{\prime} \in \mathfrak{M}(B, A)$, and

$$
m \otimes m^{\prime}=A \stackrel{\alpha}{\longleftarrow} X \longrightarrow \stackrel{\beta}{\longrightarrow} Y \stackrel{\gamma}{\longrightarrow} B \stackrel{\gamma}{\longleftarrow} Y \stackrel{\beta}{\longrightarrow} \cdots \longleftarrow X \stackrel{\alpha}{\longrightarrow} A .
$$

Starting from the middle with $P B(Y, B, Y ; 1, Y, 1)$ and repeating, shows $m \otimes m^{\prime}$ $\sim A \stackrel{\alpha}{\longleftarrow} X \stackrel{\alpha}{\rightarrow} A$; inserting $P O(A, X, A ; 1, A, 1)$ we find $m \otimes m^{\prime} \sim e_{A}$. Similarly $m^{\prime} \otimes m \sim e_{B}$ and the proof is complete.

Observe that any word

$$
A \stackrel{\alpha_{1}}{\longleftarrow} X_{1} \stackrel{f_{1}}{\longrightarrow} X_{2} \longleftarrow \cdots \longrightarrow X_{2 n-2} \stackrel{\alpha_{n}}{\longleftarrow} X_{2 n-1} \stackrel{f_{n}}{\longrightarrow} B
$$

is equivalent to

$$
\begin{aligned}
\left(A \stackrel{\alpha_{1}}{\longleftarrow} X_{1} \stackrel{1}{\longrightarrow} X_{1}\right) & \otimes\left(X_{1} \stackrel{1}{\longleftarrow} X_{1} \stackrel{f_{1}}{\longrightarrow} X_{2}\right) \otimes \cdots \\
& \otimes\left(X_{2 n-2} \stackrel{\alpha_{n}}{\longleftarrow} X_{2 n-1} \stackrel{1}{\longrightarrow} X_{2 n-1}\right) \otimes\left(X_{2 n-1} \stackrel{1}{\longleftarrow} X_{2 n-1} \stackrel{f_{n}}{\longrightarrow} B\right) .
\end{aligned}
$$

Thus, each morphism in $\mathscr{K} / \mathfrak{M}$ can be factored as $\hat{f}_{n} \circ \hat{\alpha}_{n} \circ \ldots \circ \hat{f}_{1} \circ \hat{\alpha}_{1}$ where, according to A.3, each $\hat{\alpha}_{i}$ is invertible.

The transformation $\eta: \mathscr{K} \rightarrow \mathscr{K} / \mathfrak{M}$ given by rules

$$
\begin{aligned}
& \eta(A)=A \text { on the objects, } \\
& \eta(f)=[A \stackrel{1}{\longleftarrow} A \stackrel{f}{\longrightarrow} B] \text { for each } f \in \mathscr{K}(A, B)
\end{aligned}
$$

is easily seen to be a covariant functor, called the canonical projection. Because of A.3, $\eta(\alpha)$ is invertible in $\dot{\mathscr{K}} / \mathfrak{M}$ for each $\alpha \in \mathfrak{M}$; and the known [3] universality property of $(\eta, \mathscr{K} / \mathfrak{M})$, in a formulation adapted for our purposes, is

A.4. TheOREM. Let $T: \mathscr{K} \rightarrow \mathscr{L}$ be any covariant functor to any category $\mathscr{L}$. Then

(a) There exists a covariant functor $\Delta: \mathscr{K} / \mathfrak{M} \rightarrow \mathscr{L}$ with $T=\Delta \circ \eta$ if and only if $T(\alpha)$ is invertible in $\mathscr{L}$ for each $\alpha \in \mathfrak{M}$.

(b) If $T=\Delta \circ \eta$, then $\Delta$ is unique.

Proof. (a) If $T=\Delta \circ \eta$ then, because $\eta(\alpha)$ is invertible for each $\alpha \in \mathfrak{M}$ and functors preserve invertible morphisms, $T$ has the required property. Conversely, assume $T(\alpha)$ invertible for each $\alpha \in \mathfrak{M}$. Define $\Delta$ on the objects of $\mathscr{K} / \mathfrak{M}$ by $\Delta(A)$ $=T(A)$. For each word

$$
m=A \stackrel{\alpha_{1}}{\longleftarrow} X_{1} \stackrel{f_{1}}{\longrightarrow} X_{2} \longleftarrow \cdots \stackrel{\alpha_{n}}{\longleftarrow} X_{2 n-1} \stackrel{f_{n}}{\longrightarrow} B
$$

in $\mathfrak{M}(A, B)$, let

$$
\Delta(m)=T\left(f_{n}\right) \circ T\left(\alpha_{n}\right)^{-1} \circ \ldots \circ T\left(f_{1}\right) \circ T\left(\alpha_{1}\right)^{-1} \in \mathscr{L}(\Delta(A), \Delta(B))
$$

where $T\left(\alpha_{i}\right)^{-1}$ is the inverse of $T\left(\alpha_{i}\right)$ in $\mathscr{L}$. If $m \sim m^{\prime}$, then $\Delta(m)=\Delta\left(m^{\prime}\right)$ because the operations in changing $m$ to $m^{\prime}$ are governed by commutative diagrams, and all 
morphisms "in the wrong direction" belong to $\mathfrak{M}$. Thus, $[m] \mapsto \Delta(m)$ is a welldefined $\left({ }^{15}\right)$ map $\Delta: \operatorname{hom}(A, B) \rightarrow \mathscr{L}(\Delta(A), \Delta(B))$ for each $A, B \in$ ob $(\mathscr{K} / \mathfrak{M})$ which with the above indicated correspondence of the objects, is easily seen to determine a covariant functor $\Delta: \mathscr{K} / \mathfrak{M} \rightarrow \mathscr{L}$. Clearly, $\Delta \circ \eta(A)=A$ on objects, and $\Delta \circ \eta(f)=\Delta[A \stackrel{1}{\leftarrow} A \stackrel{f}{\longrightarrow} B]=T(f)$ for each $f \in \mathscr{K}(A, B)$, so $\Delta \circ \eta=T$ and the proof is complete.

(b) Assume $T=\Delta \circ \eta=\Gamma \circ \eta$; then $\Delta(A)=T(A)=\Gamma(A)$ for each $A \in$ ob $(\mathscr{K} / \mathfrak{M})$. For each morphism of type $\hat{f}=[A \stackrel{1}{\leftrightarrow} A \stackrel{f}{\longrightarrow} B]$ we have $\Delta(\hat{f})=\Delta \circ \eta(f)=T(f)$ $=\Gamma \circ \eta(f)=\Gamma(\hat{f})$. Each morphism of type $\hat{\alpha}=[A \stackrel{\alpha}{\leftarrow} B \stackrel{1}{\longrightarrow} B]$ is invertible in $\mathscr{K} / \mathfrak{M}$, with inverse $\hat{\alpha}^{-1}=[B \stackrel{1}{\leftarrow} B \stackrel{\alpha}{\rightarrow} A]$. Thus $\Delta(\hat{\alpha}), \Gamma(\hat{\alpha})$ are invertible in $\mathscr{L}$ and, from what we have already shown, $\Delta(\hat{\alpha})=\Delta\left(\hat{\alpha}^{-1}\right)^{-1}=\Gamma\left(\hat{\alpha}^{-1}\right)^{-1}=\Gamma(\hat{\alpha})$. Since each morphism in $\mathscr{K} / \mathfrak{M}$ is representable as a composition of morphisms of these two types, we find $\Delta[m]=\Gamma[m]$ for each morphism $[m]$ and the proof is complete.

REMARK. If $\mathfrak{M}$ is the family of all morphisms in $\mathscr{K}$, then all the morphisms of $\mathscr{K} / \mathfrak{M}$ are invertible therefore each hom $(X, X)$ is a group, $\pi(X)$. Furthermore, for each $\phi \in \operatorname{hom}(X, Y)$ there is a homomorphism $\phi^{*}: \pi(X) \rightarrow \pi(Y)$ given by $\phi^{*}(f)$ $=\phi \circ f \circ \phi^{-1}$, and clearly $\phi^{*}$ is an isomorphism. Thus the groups $\pi(X)$ belonging to a "component" of $\mathscr{K}$ are all isomorphic; if $\mathscr{K}$ is "connected" then, as in topology, we can define the fundamental group $\pi(\mathscr{K})$ of a category. From C2 it follows that a covariant functor $\Phi: \mathscr{K} \rightarrow \mathscr{L}$ on "connected" categories induces a homomorphism $\pi(\mathscr{K}) \rightarrow \pi(\mathscr{L})$. It is easy to see that for $\mathrm{Top}_{0}$, the category of based topological spaces with base-point preserving continuous maps, each word from $\left(X, x_{0}\right)$ to $\left(X, x_{0}\right)$ becomes equivalent to $\left(X, x_{0}\right) \leftarrow\left(x_{0}, x_{0}\right) \rightarrow\left(X, x_{0}\right)$ and therefore that $\pi\left(\mathrm{Top}_{0}\right)=0$. Similarly, it can be shown that $\pi(\mathrm{Top})=0$.

We now show

A.5. Proposition. There exists a category $\mathscr{K}$ and a class $\mathfrak{M}$ such that $E(E(\mathfrak{M}))$ $\neq E(\mathfrak{M})$ and $E(\hat{\mathfrak{M}}) \neq E(\mathfrak{M})$, where $\hat{\mathfrak{M}}$ is the maximal stable subclass in $\mathfrak{M}$.

Proof. Consider the following diagram

$$
*_{A} \stackrel{h^{\prime}}{\longrightarrow} S_{0}^{2} \underset{\beta}{\stackrel{\alpha}{\rightleftarrows}} S_{1}^{2} \stackrel{h}{\longrightarrow} *_{B}
$$

where $S_{0}^{2}, S_{1}^{2}$ are two distinct 2-spheres, $*_{A}, *_{B}$ are distinct points, $h^{\prime}\left(*_{A}\right)=$ north pole of $S_{0}^{2}, \alpha$ is the rotation about the $z$-axis with angle $\pi$, and $\beta$ is the rotation about the same axis with angle $\pi / u, u$ an irrational. Let $\mathscr{K}$ be the category with these four distinct objects, and with morphisms generated by $h^{\prime}, \alpha, \beta, h$.

Let $\mathfrak{M}=\left\{h, h^{\prime}\right\}$; then it is immediate that $\overline{\mathfrak{M}}=$ all morphisms in $\mathscr{K}$ and $E(\mathfrak{M})$ $=\overline{\mathfrak{M}}-\mathfrak{M}$. Moreover

${ }^{\left({ }^{15}\right)}$ The set of all morphisms $A \rightarrow B$ in a category $\mathscr{L}$ will be denoted by $\mathscr{L}(A, B)$; if $\mathscr{L}=$ $\mathscr{K} / \mathfrak{M}$, this set is denoted by hom $(A, B)$. 
(a) $E(E(\mathfrak{M})) \neq E(\mathfrak{M})$. Let $\mathscr{L}$ be the smallest subcategory of Top containing $\mathscr{K}$ and the inverses $\bar{\alpha}, \bar{\beta}$ of $\alpha, \beta$. Let $\lambda: \mathscr{K} \rightarrow \mathscr{L}$ be the inclusion functor. Since $\lambda$ sends every member of $E(\mathfrak{M})$ to an isomorphism in $\mathscr{L}$, there exists (A.4) a functor $\Delta: \mathscr{K} / E(\mathfrak{M}) \rightarrow \mathscr{L}$ such that $\Delta \circ \eta=\lambda$. Now, $\alpha \notin E(E(\mathfrak{M}))$ : for if $\eta(\alpha) \eta(g)=1$ for some $g$ in $\mathscr{K}$, then $\lambda(\alpha \circ g)=1$ in $\mathscr{L}$, i.e., $\alpha \circ g=1$ in $\mathscr{L}$; however $\alpha$ does not have a right inverse in $\mathscr{K}$, so such a $g$ cannot exist in $\mathscr{K}$. Thus, $E(E(\mathfrak{M})) \neq E(\mathfrak{M})$. Moreover, since $\alpha \in E(\mathfrak{M}) \subset[E(\mathfrak{M})]^{-}$, this shows also that $E(\mathfrak{M})$ is not stable; furthermore, $\hat{\mathfrak{M}} \mathfrak{l}=\varnothing$, since $h$ and $h^{\prime}$ do not have inverses in $\mathscr{K}$, so we also have $E(\mathfrak{M}) \neq E(\mathfrak{M})$.

\section{BIBLIOGRAPHY}

1. F.-W. Bauer, Der Hurewicz-Satz, Pacific J. Math. 21 (1967), 1-14.

2. A. Dold, Partitions of unity in the theory of fibrations, Ann. of Math. 78 (1963), 223-255.

3. P. Gabriel and M. Zisman. Calculus of fractions and homotopy theory, Springer-Verlag, New York, 1967.

4. B. Mitchell, Theory of categories, Academic Press, New York, 1965.

5. P. Tulley, On regularity in Hurewicz fiber spaces, Trans. Amer. Math. Soc. 116 (1965), 126-134.

6. J. H. C. Whitehead, Combinatorial homotopy. I, Bull. Amer. Math. Soc. 55 (1949), 213-245.

RICE UNIVERSITY,

Houston, TeXas 